\title{
La condición de supervivencia en la percepción de la Naturaleza
}

\section{The survival condition in the perception of Nature}

\author{
Cerezuela Motos, Antonio \\ Universidad Politécnica de Cartagena, Departamento de Arquitectura y Tecnología de la Edificación, Universidad \\ Politécnica de Cartagena, Murcia, España. Email: cerezuela@gmx.net
}

\section{Resumen}

La aparición del informe The limits of the growth en 1972 significó entrar en una era donde la ciencia cuantifica la duración de la vida en el planeta, y cuestiona la supervivencia de la misma. Esto origina que la concienciación social re-defina la tradicional percepción cultural de una Naturaleza magnánima e ilimitada por una donde la presión por la supervivencia misma en una Naturaleza limitada, marque de manera esencial la mirada actual del siglo XXI. Para apreciar esta transformación el artículo propone un recorrido sencillo, a través de miradas concretas a lo largo de los últimos 200 años, sobre la Naturaleza y la supervivencia, que se inicia simbólicamente en la obra Caspar David Friedrich para finalizar y contrastar con las reflexiones artísticas de Eliasson Olafur. De manera complementaria se busca guiar el discurso, como testigos que muestren dicha evaluación, a través de las acepciones conocidas de supervivencia.

Palabras clave: naturaleza, supervivencia, arte, clima, arquitectura.

\begin{abstract}
The publication of the report The limits of the growth in 1972 meant to enter in an age where the science quantifies the duration of the life in the planet, and it questions the survival of the same one. Therefore, the social raising awareness re-defines the traditional cultural perception of a magnanimous and unlimited Nature for one where the pressure for the survival itself in a limited Nature, marks in an essential way the current view in the 21st century. This article shows this transformation through a simple path, across relevant viewpoints in last 200 years, which relate Nature and survival. It begins symbolically in the work Caspar David Friedrich to finish and to contrast with Eliasson Olafur's artistic reflections. Complementary, it supports to clarify the above mentioned change, with different and known meanings of survival.
\end{abstract}

Key words: nature, survival, art, climate, architecture. 


\section{Introducción}

Desde la necesidad de una nueva conciencia sobre nuestro medioambiente, proclamada ya desde el año 1987 con el Informe Brundtland (1) la relación del hombre con la naturaleza está sometida a nuevas revisiones. El cambio climático marca un antes y después en las sensibilidades al respecto del medioambiente. La constatación científica de que nuestro mundo con la actividad humana sobre el mismo es un mundo finito, la mera posibilidad de que la razón humana posibilite calcular la fecha de su propia desaparición, como intentó mostrar en 1972 el informe The Limits of Growth (2) , es una cima inédita alcanzada por la ciencia de gran calado e importantes consecuencias.

Por tanto, la consideración de un mundo finito y amenazado con desaparecer no deja indiferente a la sociedad, y ésta ha de asimilar y reaccionar ante las nuevas circunstancias. Es entonces cuando la cuestión de la supervivencia misma se constituye en un eje relevante para replantear muchas de nuestras actividades, y sobre todo la que se plantea en este artículo, la percepción de la Naturaleza y la manera de posicionarnos en nuestro entorno natural, cuya relación será estudiada a través de la noción de supervivencia como elemento conductor.

Estas nuevas relaciones deberían conducir a determinados planteamientos para la arquitectura, que sería el objetivo final en pasos posteriores. Como dice Josep María Montaner (3), la teoría de la arquitectura se analiza desde dos premisas, que "cada posición formal remite a una concepción del mundo", y que los "métodos de interpretación deben ser cada vez más complejos". Por tanto el análisis de esa concepción del mundo desde esa mirada de la Naturaleza limitada y amenazada de la que depende nuestra supervivencia, nos aportaría posiciones y planteamientos en la arquitectura.

Para poder obtener una valoración de estas nuevas relaciones entre naturaleza y supervivencia, se establecen varios elementos que acoten y parametricen el campo de estudio: un marco histórico retrospectivo, un compendio de referencias de diversos ámbitos (artísticos, científicos, arquitectónicos) que sea representativas de esa relación naturaleza-supervivencia, y una definición oficial de supervivencia.

El marco histórico tiene su inicio simbólicamente en la obra de Caspar David Friedrich, que casualmente coincide con en el inicio, según algunos científicos, con el ciclo del Antropoceno sobre el año 1700.

Por otra parte, tomaremos como referencia la definición de supervivencia, de la Real Academia Española (RAE), la cual remite a la acción y efecto de sobrevivir compuesta por tres acepciones que se a continuación se transcriben:

1. "Dicho de una persona: Vivir después de la muerte de otra o después de un determinado suceso.

2. Vivir con escasos medios o en condiciones adversas.

3. Dicho de una persona o de una cosa: Permanecer en el tiempo, perdurar".

La primera se refiere directamente a la posibilidad de eludir la propia muerte, la segunda es la propia acción de enfrentarse a la adversidad que amenaza nuestra vida y la tercera es la apropiación más abstracta y etérea de superar la muerte.

\section{Lo sublime en la Naturaleza}

La primera referencia que tomamos es la obra del pintor Caspar David Friedrich, por su carácter representativo del periodo Romántico, en el que la Naturaleza se aprecia desde el asombro y el sobrecogimiento de una fuerza vital ilimitada, inmensa e infinita. La Naturaleza y sus enigmas es el centro de una cultura atraída por el "abismo deseado" [ Rafael Argullol (4) ] y es la impotencia de lo racional para abarcar lo que le acontece, lo que conduce a que se la emoción lo que guía al individuo, en busca de la experiencia más intensa que sobrecoge al alma, esto es, en busca de lo sublime en la Naturaleza.

La travesía simbólica del Caminante que se enfrenta a las adversidades de la Naturaleza alimenta el espíritu en busca de la eternidad ansiada que representa la fuerza superior que es la Naturaleza. 


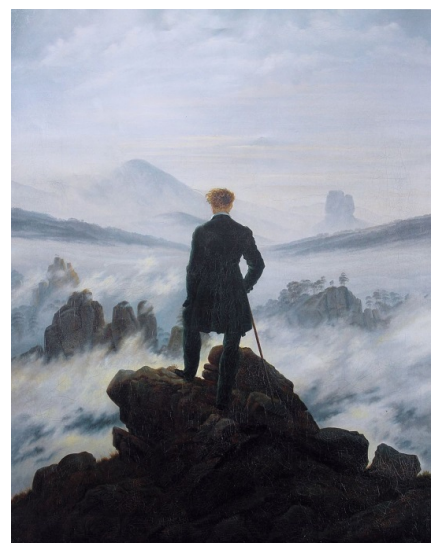

Fig: 1 "Der Wanderer über dem Nebelmeer" Caspar David Friedrich (1818).

Esta percepción del entorno natural que comparte el Pintoresquismo en arquitectura, coetáneo del Romanticismo, constituye el inicio del recorrido histórico propuesto, y que podemos clasificar en la tercera acepción que nos ofrece la RAE, la que plantea la supervivencia desde la permanencia en el tiempo, entendida en este caso como la aspiración humana de perdurar, y que para los romanticistas representa el mayor de los anhelos, alcanzar la eternidad.

\section{La Naturaleza maestra en supervivencia}

La siguiente referencia tiene una cercanía histórica con nuestro momento, y coincide en los años de la postguerra, cuando todavía los movimientos ecologistas no se habían constituido en una fuerza presente en la sociedad, y encontramos a Alejandro de la Sota, en el año 1956, divagando sobre Arquitectura y Naturaleza de la siguiente manera:

"La Naturaleza, en su profundo, es inmutable. Basta alejarse de ella, subir a una altura y ver que realmente aguanta el ataque del tiempo, de los siglos, de los milenios: la Naturaleza se sobrevive"

“(..) Crea el artista obras que le sobreviven, crea para sobrevivirse, porque no es sobrevivir la permanencia después de muerto, es el sostenimiento de la vida; solamente la Obra de Arte lo consigue. Crear ("producir algo de la nada") por antonomasia es solamente a Dios a quien pertenece; en su acercamiento, el hombre crea, tanto más elevado será su arte cuanto más elevado sea este acercamiento. Unida a esta elevación, a esta pureza en la concepción, va la supervivencia de la Obra de Arte; su valor se mide por su supervivencia. No es necesaria la sobrevivencia de la obra en sí; basta con que exista en la idea que alentó su creación". (5)

Esta percepción de Alejandro de la Sota sobre la inmutabilidad de la Naturaleza como algo que sobrecoge, conecta con la permanencia en el tiempo en una entidad superior anhelada de los artistas románticos, anteriormente referenciada.

Pero Alejandro de la Sota abandona el plano espiritual, para conducir esa divagación hacia obtener una enseñanza de la maestra Naturaleza. Con una visión racional y desde la sencillez podemos aprender lecciones de belleza funcional ligada a la supervivencia en la Naturaleza, inspiradora de formas y material.

Este texto de Alejandro de la Sota es interpretado por José Manuel López-Peláez (6) en un artículo titulado Arquitectura y abstracción, y dice al respecto: "para Alejandro de la Sota la Naturaleza es una energía, una fuente de inspiración que puede ser traducida a símbolos y sugerir metáforas muy cercanas a las ideas de arquitectura". Es decir, supervivencia sería es fuerza vital manifiesta en la Naturaleza y que proporciona claves fundamentales para construir nuestro entorno físico.

Tal como nos recuerda López Peláez para de la Sota "el legado de la Arquitectura no son huellas sino ideas". Es decir lo que traspasa los límites cronológicos no es la presencia física sin "la idea que alentó su creación". LópezPeláez explica como al hablar Sota de Arquitectura y Naturaleza como dos categorías y polaridades contrapuestas, que pueden asimilarse a Abstracción y Naturaleza, situando la abstracción y la arquitectura en el mismo lado. Esto es porque Sota entiende que para aprender con inteligencia de la Naturaleza es necesaria la mirada abstracta. 


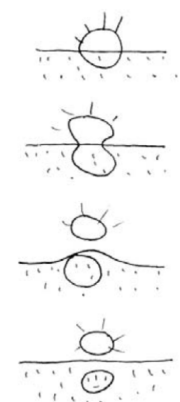

Fig: 2 “. Ideograma de la Casa Domínguez.

Alejandro de la Sota (1976).

Años más tarde Sota concebiría varias casas, la Casa Domínguez o la Casa Guzmán, en base a la idea abstracta de la relación con la Naturaleza, en que las estancias del dormir, reposo y descanso, cerca de la muerte, deberían estar en el subsuelo en la tierra, y conforme la actividad del pensamiento es más intenso las estancias asciende, hasta situarse sobre la cota cero (fig. 2).

Esta posición de supervivencia de Sota tiene que ver con una disposición inteligente en el entorno, donde la Naturaleza, maestra en supervivencia provee de recursos abstractos y formales para perdurar en un entorno adverso. Por ello estaría más cerca de la segunda acepción de la RAE.

\section{El refugio bioclimático}

En el año 1963 Victor Olgyay (7) publica en la Universidad de Princeton (Estados Unidos) un manual en el que pone en relación el diseño arquitectónico en función del clima. Tal como explica Rafael Sierra en la introducción de la versión española (Gustavo Gili, 1998), Olgyay ideo el término bioclimático, no como una manera de adjetivar la arquitectura, tal como ha ocurrido a posteriori, si no se hablaba de "interpretación bioclimática" (bioclimatic approach) de la arquitectura definiendo los efectos del clima sobre el hombre (el "bios").

La supervivencia en la Naturaleza ocupa también en Olgyay la prioridad absoluta, y está relacionada con una visión antropocéntrica del clima, es decir, partiendo de las condiciones físicas y sicológicas que definen la zona de confort del hombre se establecen los límites admisibles de vida, los cuales si se sobrepasasen no sería posible la supervivencia humana. Ese perímetro vital queda definido en sus cartas bioclimáticas, que resulta ser la intersección cuantitativa de variables características que se interrelacionan, como la temperatura, la humedad, la radiación o la velocidad del viento.

La carta bioclimática (fig.3) resulta pues, ser un mapa de las posibilidades de la vida biológica del hombre en la Naturaleza. Incluso Olgyay llega a ampliar el ámbito de estudio a la relación entre el clima, la herencia racial y el desarrollo cultural de los pueblos, lo que muestra la profundidad de sus estudios sobre la supervivencia física en la Naturaleza, en la que la arquitectura tendría el papel de protector ante los condicionantes del clima. Es por todo ello que su trabajo se fija en las construcciones de los animales para defenderse de la intemperie, en su condición de refugio adaptado al clima, esto constituye una inspiración para la "reconciliación intuitiva con las fuerzas de la naturaleza".

Muy cercano a Sota, Olgyay se posiciona ante la Naturaleza para obtener referencias y modelos que aplicar a la arquitectura, para resolver las necesidades biológicas del cuerpo humano de supervivencia en el entorno natural con respecto al clima, donde el ingenio humano y el uso de sus recursos técnicos responderían a las adversidades para subsistir. En este sentido se acercaría como Sota a la segunda acepción de la RAE.

En ambas percepciones es la razón la herramienta de acercamiento a la Naturaleza, la que facilitaría soluciones dentro de la cultura tecnológica de la postguerra a la que pertenecen, en contraposición con el romanticismo, donde la aspiración del alma es transcender ante la obra de la Naturaleza. No obstante, en Sota expresa su emoción ante fuerza de la Naturaleza, parecida a la de Friedrich, y al hablar de las ideas como ente que perdura en el tiempo, 
se muestra menos científico que Olgyay, que es pionero en dotar de un método científicamente la adecuación de la arquitectura al clima.

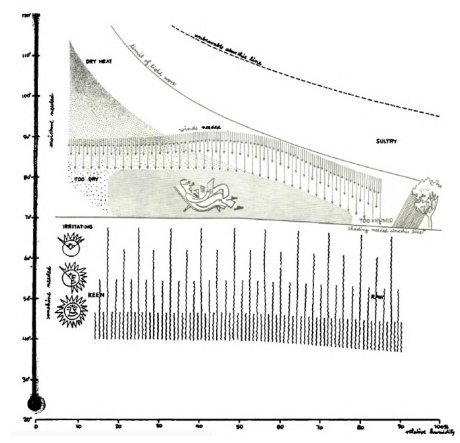

Fig: 3. Carta bioclimática. Victor Olgyay

\section{La supervivencia auditada}

El término Antropoceno, según diversas fuentes, fue acuñado en el año 2000 por el premio Nobel Paul Crutzan, Vicepresidente del Programa Internacional de Geosfera y Bioesfera, para dar nombre a nuevo ciclo geológico de la Tierra en el cual la actividad humana es el factor determinante en la transformación de los ecosistemas vitales del planeta. El propio Crutzen (8) sitúa el comienzo de esta época en la revolución industrial, a partir del 1700, cuando se inicia a nivel global el incremento de dióxido de carbono en la atmosfera.

Tras la segunda guerra mundial tiene lugar una paulatina mayor concienciación social sobre las perturbaciones antrópicas sobre el medioambiente, hasta que en el año 1972 el Club de Roma (organización no gubernamental fundada en 1968) publica el informe The Limits to Growth donde expertos en medioambiente advierten que existen suficientes indicadores como el incremento de la población mundial, la industrialización o la explotación de los recursos naturales, para que en un corto periodo, se estimó en 100 años, se agotaran los recursos naturales lo que conduciría la extinción de la vida en la Tierra.

En el año 2015 la revista The Anthropocene Review publica el informe elaborado por expertos científicos titulado The trajectory of the Anthropocene: The Great Acceleration (9), que actualiza otro anterior del año 2004, en él se muestra los datos numéricos del fenómeno denominado La gran aceleración, en el que se muestra como tras la segunda guerra mundial la actividad humana incrementa exponencialmente aumentado igualmente los datos de agotamiento medioambiental del planeta. En la fig. 4 que gráficamente representando con exactitud el fenómeno en base a diverso indicadores.

En definitiva, desde la publicación de The Limits to Growth, se sucede como algo habitual en nuestra cultura contemporánea la emisión de informes, suscritos por expertos diferentes en diversos campos de la ciencia, que dibujan unos escenarios de futuro incompatibles con la vida. Todo ello, tras el impacto y difusión de las conclusiones de dichos informes, dota en la conciencia colectiva de la sociedad contemporánea, de una gran relevancia la condición de supervivencia en la percepción del hábitat en que vivimos.

Los informes que estudian el Antropoceno, dibujan un paisaje de degradación continua de las constantes vitales del planeta, y sustituye al hombre como factor determinante en el devenir de la Tierra. Los expertos sitúan el inicio de este ciclo geológico alrededor de 1700, época representada en este artículo con el momento cultural de Friedrich, y sitúan en la postguerra el inicio de la "Gran aceleración", momento representado en este estudio por las posiciones de Sota y Olgyay.

Desde The Limits to Growth, la ciencia y sus herramientas, constituidas por indicadores que monitorizan y parametrizan las constantes vitales del planeta, constituirá un referente cultural para la sociedad y los poderes políticos. El alcance de los estudios científicos, aunque sean refutados por otras partes de la ciencia, serán lo centro de preocupación y acción por parte de la ciudadanía. Los datos que continuamente trasladan al ámbito público confiere a la supervivencia tintes dramáticos, más cercanos a la primera acepción de la RAE. 


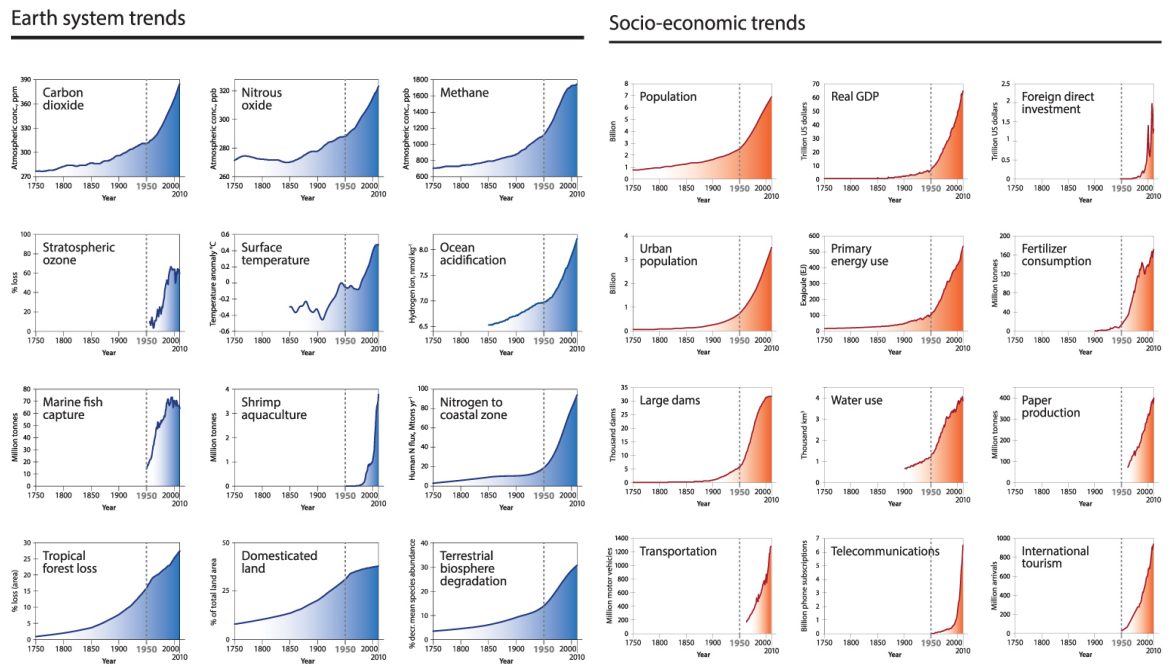

Fig: 4. "The Great Aceleration" (2015),

The Anthropocene Review.

\section{La desaparición de la Naturaleza}

En el año 1997 tiene lugar la cumbre mundial que dio lugar al Protocolo de Kyoto por el que activaron instrumentos legislativos en los países más industrializados. La gravedad de la situación y las demandas sociales e nivel planetario requerían exigía a los gobiernos de occidente la aplicación inmediata de medidas para paliar los efectos destructivos de la actividad humana sobre el medioambiente.

Los artistas Olafur Eliassen y Minik Rosing, con ocasión de diferentes cumbres sobre el cambio climático, primero en Copenhague (2014) (fig.5) y después en Paris (2015), instalaron en la Plaza del Ayuntamiento y en la Plaza del Panteón respectivamente, y 12 grandes bloques traídos de Groenlandia para concienciar a la sociedad sobre el cambio climático. El objetivo era ofrecer la experiencia tangible y real del deshielo del ártico en ubicaciones tan lejanas como capitales europeas. Durante día y noche los ciudadanos podían apreciar, tocar e interactuar con estas moles de hielo que desaparecían día tras día. Los artistas consiguieron de este modo proporcionar una imagen, asociada a la sensación de supervivencia, que iba más allá de los datos científicos reflejados en concienzudos gráficos.

De nuevo el arte es reflejo de las preocupaciones de su época, y presenciamos un salto cultural de las pinturas de Friedrich, en concreto la obra Eismeer (1824), a el Eis watch de Eliasson. Pasamos de ser observadores de la grandeza de una Naturaleza ilimitada y eterna, a ser testigos de parte de una Naturaleza que desaparece entre nosotros.

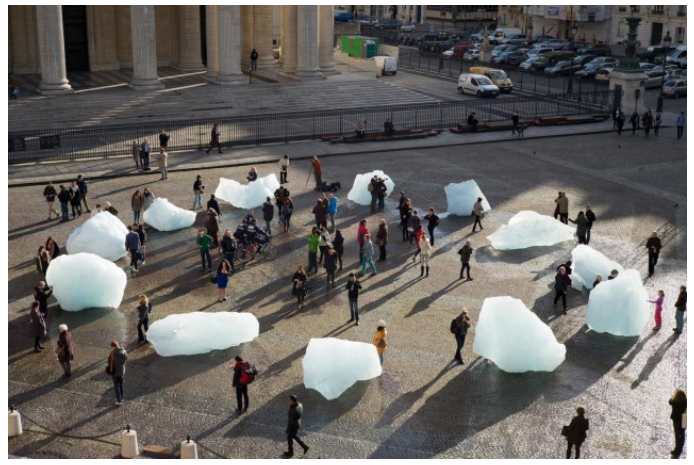

Fig: 2. "Eis watch", Eliasson Olafur (2014) 
Por otra parte, los avances tecnológicos han amplificado la capacidad de la ciencia para enviar mensajes sobre el estado del planeta. La capacidad de analizar, medir y profundizar en los más diversos aspectos de loa que acontecen en la corteza terrestre ha facilitado la producción de nuevas imágenes de escala global o planetaria que calan en la cultura actual La ciencia lidera las explicaciones a escala global de nuestro entorno, incluido los aspectos más preocupantes como las alteraciones de las condiciones medioambientales.

En este sentido la imagen de la fig. 6, tomada por satélite, es una mirada científica del siglo XXI, que completaría la serie de Friedrich y Eliassen, donde la Naturaleza es auditada, en tanto la ciencia es capaz de medir los movimientos diarios de los bloques de hielo en el lago Baikal de Siberia, así como su edad, volumen, etc. Pero el mensaje del científico, que domina en muestra cultura, no es la magnanimidad de la Naturaleza, si no su condición de objeto limitado que tiende a la desaparición, tal como Eliassen quería mostrarnos.

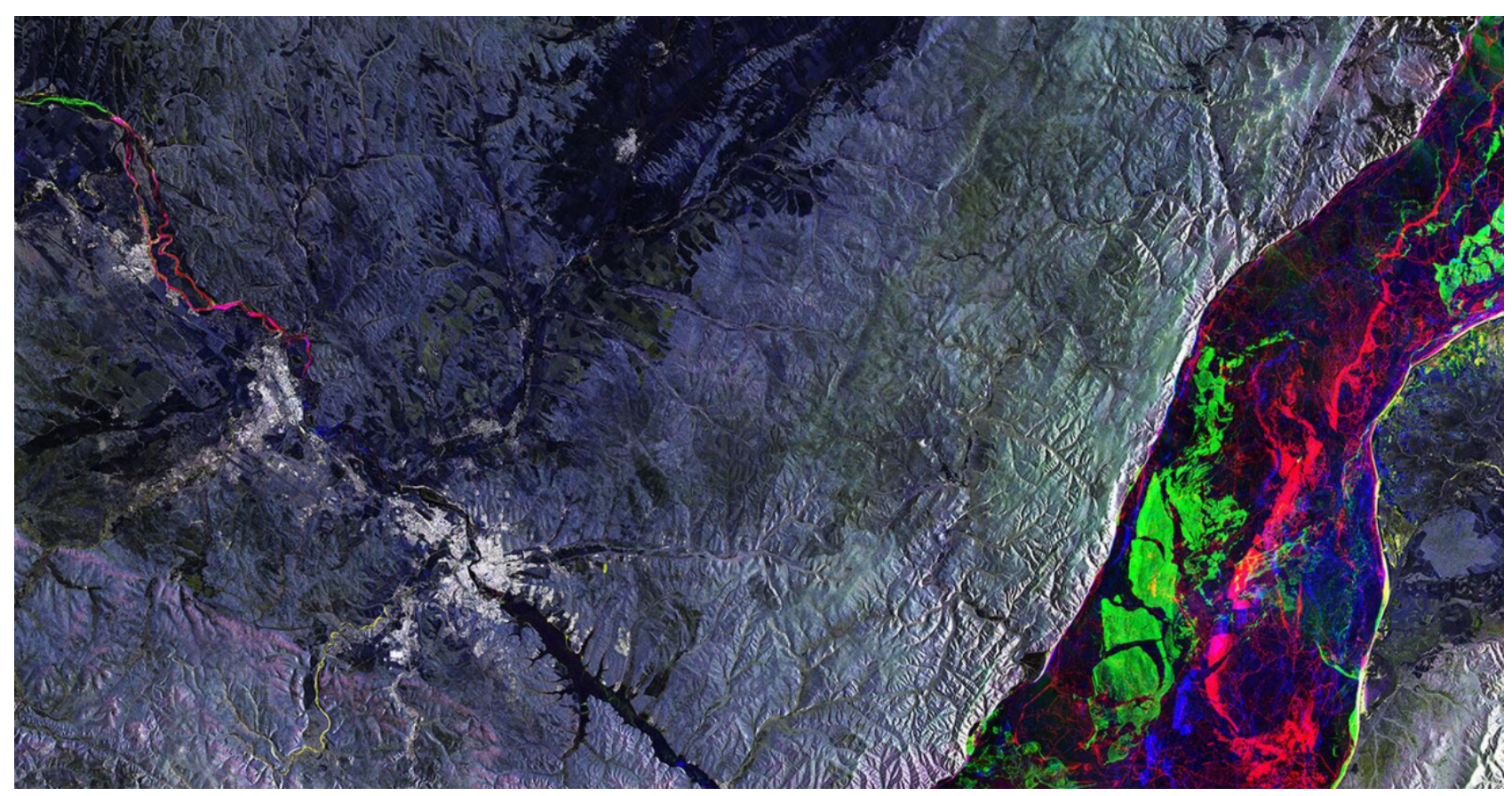

Fig: 6. Imágenes Sentinel-1A(2015). ESA.

Medición de los bloques de hielo en Siberia.

Los colores muestras los movimientos diarios, así el volumen y la edad de los bloques de hielo.

En este sentido la artista Bárbara Fluxa (10) , en la obra "Mapa de un lugar desaparecido" (fig. 7), va más allá, en el año 2010 la cartografía con tecnología avanzada utilizado por científicos las huellas de un pueblo sumergido en el embalse de la Almendra en Zamora. Fluxá realiza una batimetría con un Sonar ecoacústico y consigue un modelado informático que reproduce la traza urbana del pueblo bajo las aguas.

La obra de Fluxá tiene su complemento en el vuelo aéreo de 1957 para contrastar coincidencias. La artista diluye en su obra los procedimientos y sistemas que separan de la ciencia y el arte. Fluxá explica que lo que aparece es "una cartografía simbólica en la que lo temporal distorsiona la realidad objetiva". Pero entre la acción de la figura 6 y 7 , realizadas, la primera por científicos y la segunda por la artista, no existe diferencias en la metodología y uso de las herramientas.

En el trasfondo aparece la reflexión sobre el tiempo, lo que permanece, lo que desaparece, lo que es de la naturaleza y lo que es de la acción del hombre, todo representado de manera simbólica. La acción de Fluxá logra décadas después, hacer sobrevivir el pueblo desaparecido mediante una imagen tecnológica del siglo XXI. LA cuestión de la desaparición y la eliminación del entorno sitúan todas estas obras en la primera acepción de la RAE. 


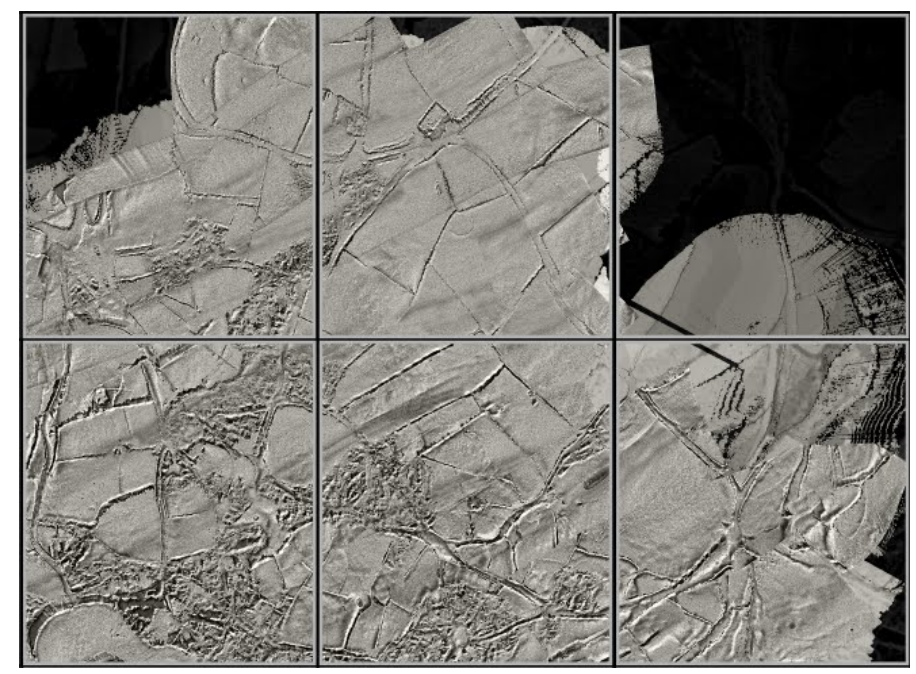

Fig: 7. "Mapa de un lugar desaparecido". Bárbara Fluxá, (2011).

\section{Reflexión y conclusiones}

\#1. Vivir después de la muerte

"Ciencia

Tecnología

Cuantificación

\#2. Vivir con escasos medios o en condiciones adversas

"Abstracción

Mímesis

Simbiosis
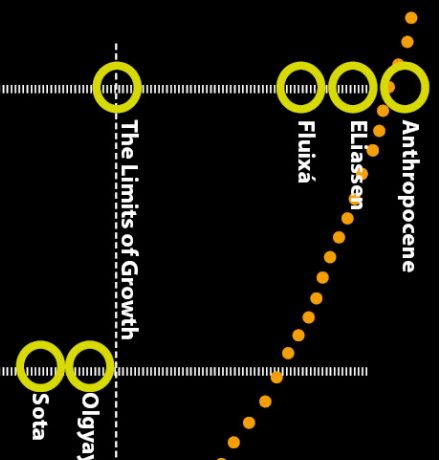

\#3. Permanecer en el tiempo

Sobrecogimiento Emoción

Enternidad

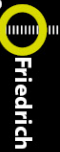

Fig: 8. "Mapa de la supervivencia".

Antonio Cerezuela, (2017).

To cite this article: La condición de supervivencia en la percepción de la naturaleza.

Antonio Cerezuela. https://doi.org/10.14198/i2.2017.5.02 
Una vez finalizado el recorrido histórico, realizamos para concluir una breve recapitulación del mismo. Hemos iniciado la andadura con el Caminante de Friedrich en los albores del Antropoceno, fechado en 1700 según los geólogos de nuestra época, cuestión que en su momento ignoraban en el Romanticismo. Tras ello saltamos a los años de la posguerra, donde los expertos actuales han fechado el inicio de la Gran Aceleración, que Sota y Olgyay presenciaban pero que desconocían el alcance real de sus consecuencias.

Para terminar entramos en el periodo que marca nuestra contemporaneidad, la que situamos el informe The limits of the growth en 1972 , de gran difusión social, como inicio de una época donde la cuantificación de los problemas medioambientales determinará una nueva forma de apreciar la Naturaleza. En este tramo histórico se sucede la publicación de informes que conducen a la ciencia a tomar una posición de gran relevancia desplazando otras sensibilidades. La ciencia, o parte de ella, ponen de relieve la fragilidad del medio ambiente que nos rodea, en constante amenazada, por lo que la cuestión supervivencia entendida como desaparición o aniquilación de la vida, es una idea que persiste desde entonces hasta nuestros días.

Este artículo tiene muestra cómo ha evolucionado el significado supervivencia, desde lo transcendental que atañe el alma y a lo etéreo hasta la cuestión más física y material de la vida. Tras doscientos años los datos científicos dominan sobre cualquier otro factor, sobre la conciencia colectiva de la supervivencia. La ciencia y la tecnología han configurado una precepción de la Naturaleza donde sobrevivir, desaparecer y controlar lo natural, han influido en la mirada de nuestra época, tal como numerosos artistas nos muestra utilizando las tecnologías al modo de la ciencia.

Las relaciones entre arte, ciencia y supervivencia, traen cuestiones culturales que marcan la época, que pasan de la razón a la emoción, de lo etéreo a lo físico, con el consiguiente reflejo en la arquitectura, como se indicaba al inicio de este artículo, cuando Montaner relaciona la concepción del mundo con las formas arquitectónicas.

De alguna manera ya ocurre cuando los proyectos de arquitectura se miden su sostenibilidad por la cuantificación de determinado parámetros, y no por sus discursos de simbiosis con la Naturaleza, por ejemplo. Pero si admitimos el papel preponderante de los resultados científicos, también cabría radicalmente plantearnos como solución no edificar por prescripción científica. De una forma u otra, los geólogos, los biólogos, entre otros, son los que, a través de sus avances y recursos tecnológicos, está consolidando la noción de supervivencia en nuestra mirada de la Naturaleza.

\section{Notas bibliográficas}

1. Informe: Our Common Future, From One Earth to One World. Oslo : World Commission on Environment and Development, 1987.

2. MEADOWS, Donella H., MEADOWS, Dennis. The Limits to Growth: A Report for the Club of Rome's Project on the Predicament of Mankind. . New York : Universe Pub, 1972.

3. MONTANER, Josep María. Sistemas arquitectónicos contempráneos. Barcelona : Gustavo Gili, 2008.

4. ARGULLO, Rafael. La atracción del abismo. Madrid : El Alcantilado, 1993.

5. SOTA, Alejandro de la Sota. Arquitectura y Naturaleza. [aut. libro] Ginés Garrido y Andrés Cánovas. Textos de crítica de arquitectura. Madrid : Departamento de proyectos de la ETSAM, 2003, pág. 255.

6. LÓPEZ-PELÁEZ, José Manuel. Arquitectura y abstracción. [aut. libro] Ginés Garrido y Andrés Cánovas. Textos de crítica de arquitectura. Madrid : Departamento de proyectos de la ETSAM, 2003, pág. 263.

7. OLGYAY, Victor. Design with Climate. Bioclimatic approach to arquitectural regionalism. Princenton : Princeton University Press, 1963.

8. The Encyclopedia of Earth. [En línea] [Citado el: 24 de mayo de 2017.] http://editors.eol.org/eoearth/wiki/Anthropocene.

9. The trajectory of the Anthropocene: The great acceleration. WILL Steffen, et al. 1, Liverpool : The Anthropcene Review, 2015, Vol. 2, págs. 81-98. 
10. BURKE, Edmund. Indagación filosófica sobre el origen de nuestras ideas acerca de lo sublime y de lo bello. Madrid : Alianza Editorial, 2005.

11. FLUXÁ, Bárbara. "Mapa de un lugar desaparecido". [En línea] 2011. [Citado el: 2017 de 5 de 20.$]$ http://barbarafluxa.blogspot.com.es/.

12. Ecología Politica. [En línea] 1991. [Citado el: 27 de mayo de 2017.] www.ecologiapolitica.info.

13. KANT, Inmanuel. Observaciones sobre lo bello y lo sublime. Madrid : Fondo de Cultura Económica de España, 2005.

14. KUHN, Thomas S. La estructura de la revoliciones científicas. Madrid : Fondo de Cultura Económica de España, 2006.

15. MONTANER, Josep María. Belleza de las arquitecturas ecológicas. La Modernidad superada. Barcelona : Gustavo Gili, 2011.

16. Arquitectura: Cambio de Clima. Madrid : Fundación Arquitectura y Clima, Arquitectura Viva, 2016.

\section{Biografía}

Antonio Cerezuela Motos. Profesor de Proyectos Arquitectónicos en la ETSAE, Universidad Politécnica de Cartagena. Diploma Estudios Avanzados por la ETSAM (2011). Coordinador área técnica del CSCAE (2006-2016). Colaborador servicios técnicos COAMU (2001-2005). 